\title{
An association between arterial pulse pressure and variation in the fibrillin-1 gene
}

\author{
J T Powell, R J Turner, A M Henney, G J Miller, S E Humphries
}

Cardiovascular and Pulmonary Research Division, Charing Cross and Westminster Medical School, Fulham Palace Road, London W6 8RF, UK $\mathrm{J}$ T Powell

R J Turner

Division of Cardiovascular Genetics, Rayne Institute, UCL Medical School, London WC1, UK

A M Henney

S E Humphries

Wolfson Institute of Preventative Medicine, The Medical College of St Bartholomew's Hospital,

Charterhouse Square, London EC1, UK

G J Miller

Correspondence to: Dr Powell.

Accepted for publication 24 June 1997

\begin{abstract}
Objective-To investigate whether variation in the fibrillin-1 gene was associated with blood pressure in healthy middle aged men, as had been observed in patients with abdominal aortic aneurysm. Design, setting, and patients-Middle aged men $(n=245)$, aged 50 to 61 years, were recruited from one of the nine general practices participating in the second Northwick Park heart study. Blood samples were obtained for the preparation of genomic DNA and analysis of plasma variables.

Main outcome measures-Systolic, diastolic, and pulse pressures; fibrillin-1 genotype characterised with a four allele variable tandem nucleotide repeat polymorphism in intron 28.

Results-In healthy middle aged men only three common genotypes were observed: 2-2 (frequency $54.1 \%), 2-3(16 \%)$, and $2-4$ $(15 \%)$. The mean arterial systolic (and pulse) pressure varied according to fibrillin-1 genotype: 2-4 genotype, 126.3 (47.6) mm Hg; 2-2 genotype, 131.0 (51.3) mm Hg; and 2-3 genotype, 135.5 (54.2) mm Hg. The median pulse pressure was $50 \mathrm{~mm} \mathrm{Hg}$. Distribution of men around the median pulse pressure, according to genotype, showed a significant trend for patients of 2-4 genotype to have the lowest pulse pressures, those of 2-2 genotype to have intermediate pressures, and those of 2-3 genotype to have the highest pulse pressures ( $p=0.003$ for healthy men). Conclusions-There appears to be a significant association between fibrillin-1 genotype and arterial pulse pressure in men aged 50 to 61 years.

(Heart 1997;78:396-398)
\end{abstract}

Keywords: blood pressure; fibrillin; pulse pressure

The genetic contribution to pathological elevation of blood pressure has been demonstrated most clearly in rats. ${ }^{12}$ In particular, it has been shown that independent genetic loci influence different haemodynamic components of blood pressure, separate loci being associated with diastolic blood pressure and pulse pressure in Lyon hypertensive rats. ${ }^{1}$

Mechanisms associated with the elevation of pulse pressure include the increased stiffness of the aorta and modulation of the elastic properties or calibre of the aorta and other vessels which influence the propagation of the arterial pulse. The distensibility of the aorta depends upon the concentration of elastin and other elastic microfibril proteins, including fibrillin. With aging the aorta becomes stiffer, aortic elastin concentration is reduced, and there is a tendency for blood pressure to increase. ${ }^{3-5}$ Apart from these age dependent changes, there may be a genetic contribution to human arterial pulse pressure which results from variation in the genes encoding the proteins of the elastic matrix of the aorta.

A study of genetic polymorphisms in these matrix proteins in patients with abdominal aortic aneurysm had suggested that both systolic blood pressure and pulse pressure were associated with variation in the Marfan gene fibrillin- $1 .{ }^{6}$

Here we have investigated the hypothesis that in healthy middle aged men polymorphic variation in the fibrillin-1 gene is associated with either systolic blood pressure or aortic pulse pressure. For this purpose we have used a tandem nucleotide repeat polymorphism in intron 28 (non-coding) of the fibrillin-1 gene. This is the same polymorphism used to map the Marfan gene (fibrillin-1) to chromosome $15 .^{78}$ the different alleles of this polymorphic locus are fragments obtained from intron 28 of the fibrillin-1 gene defined in terms of its length, since this intron contains a variable number of repeats of the sequence TAAAA, linked in tandem. A sample of fragments from a population of individuals shows substantial variation in length because of variation in the number of repeats. These variable nucleotide tandem repeats (VNTR) are common in the human genome and are used widely in linkage analysis and population genetics. ${ }^{9}$ In this study we have used the (TAAAA) $)_{n}$ repeat in the fibrillin-1 gene as a polymorphic marker, demonstrating the presence of four alleles, but only three common genotypes.

\section{Methods}

In 1991 healthy middle aged men of Caucasian origin $(n=245)$, between 50 and 61 years of age, were recruited from one of the nine general practices participating in the second Northwick Park heart study. ${ }^{10}$ The baseline population was screened for eligibility and men were excluded for one or more of the following: regular medication with aspirin or anticoagulants, malignancy (except skin cancer other than melanoma), cerebrovascular disease, history of unstable angina or myocardial infarction, electrocardiographic appearances suggestive of a previous myocardial infarct, diseases exposing staff to risk of infection, and mental disorder or other conditions precluding 


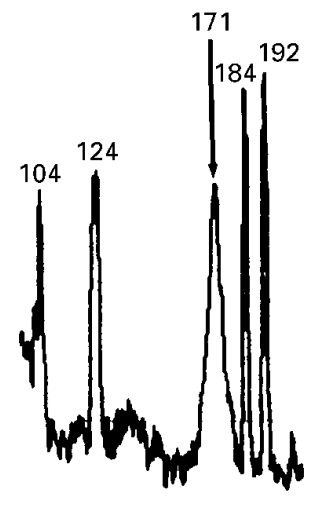

A 2-2 genotype

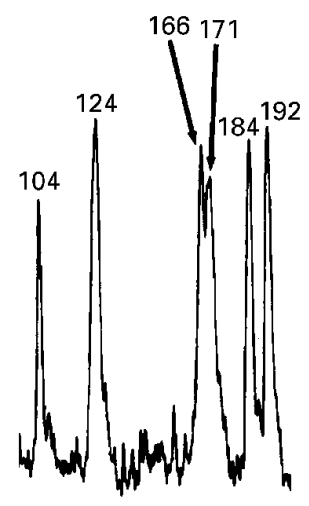

B 2-3 genotype

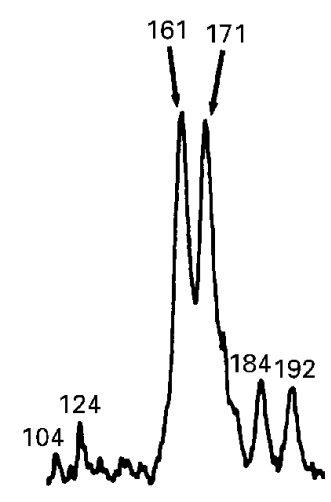

C 2-4 genotype
Figure 1 The use of capillary electrophoresis to analyse the TAAAA repeat in intron 28 of the fibrillin-1 gene. The three most common genotypes 2-2 (171 bp), 2-3 (166, $171 \mathrm{bp})$, and 2-4 (161 and $171 \mathrm{bp}$ ) are shown, flanked by markers at 104, 124, 184, and $192 \mathrm{bp}$.

informed consent or regular attendance for follow up examinations. In all $70 \%$ of the baseline population were eligible for this study. Men were interviewed about their smoking habits and current medication. The mean (SD) age of these men was 54.4 (3.0) years, body mass index was $26.3(3.2) \mathrm{kg} / \mathrm{m}^{2}$, and $38 \%$ were current smokers.

Resting blood pressure was determined in the left arm, after the subject or patient had been seated for five minutes, by an observer trained in blood pressure recording using a random zero sphygmomanometer. Subjects and patients had not smoked in the preceding hour. Blood samples were obtained to provide plasma for the measurement of cholesterol, and cells for the isolation of genomic DNA, according to routine protocols. DNA samples were genotyped for a (TAAAA) $n$ repeat in the fibrillin-1 gene on chromosome 15 using the primers previously described to amplify the TAAAA repeat in intron $28 .^{7}$ Samples were genotyped by researchers who were blinded to the blood pressures and baseline or follow up drug treatment of the 245 men. The polymerase chain reaction was performed in 96-well

Table 1 Distribution of fibrillin-1 genotypes according to blood pressure (healthy middle aged men)

\begin{tabular}{lccccl}
\hline Blood pressure $(\mathrm{mm} \mathrm{Hg})$ & $2-2(n=154)$ & $2-3(n=34)$ & $2-4(n=33)$ & p value \\
\hline Pulse pressure < 50 & $84(54.4 \%)$ & $10(29.4 \%)$ & $23(69.7 \%)$ & \\
Pulse pressure $>50$ & $70(45.5 \%)$ & $24(70.6 \%)$ & $10(30.3 \%)$ & 0.003 \\
Mean pulse pressure & $51.3(12.0)$ & $54.2(13.2)$ & $47.6(11.4)$ & 0.08 \\
Systolic pressure < 131 & $81(52.6 \%)$ & $14(41.2 \%)$ & $19(57.6 \%)$ & \\
Systolic pressure > 131 & $73(47.4 \%)$ & $20(58.8 \%)$ & $14(42.4 \%)$ & 0.37 \\
Mean systolic pressure & $131.0(18.0)$ & $135.5(16.7)$ & $126.3(15.4)$ & 0.10 \\
Diastolic pressure < 80 & $79(51.3 \%)$ & $15(44.1 \%)$ & $17(51.5 \%)$ & \\
Diastolic pressure > 80 & $75(48.7 \%)$ & $19(55.9 \%)$ & $16(48.5 \%)$ & 0.74 \\
Mean diastolic pressure & $79.7(10.4)$ & $81.3(10.4)$ & $78.7(9.8)$ & 0.58 \\
\hline
\end{tabular}

The distribution around the median is shown, followed by mean (SD). $\mathrm{p}$ values are for $\chi^{2}$ (distributions) or analysis of variance (means).

Table 2 Other characteristics according to fibrillin-1 genotype (healthy middle aged men). Values are mean (SD)

\begin{tabular}{lcccc}
\hline & $2-2(n=154)$ & $2-3(n=34)$ & $2-4(n=33)$ & $p$ value \\
\hline Age $($ years $)$ & $54.4(3.0)$ & $54.7(2.9)$ & $53.7(3.1)$ & 0.34 \\
BMI $\left(\mathrm{kg} / \mathrm{m}^{2}\right)$ & $26.3(3.2)$ & $26.0(2.8)$ & $26.4(3.3)$ & 0.84 \\
Cholesterol (mmol/1) & $5.7(1.0)$ & $5.8(1.0)$ & $5.7(1.0)$ & 0.89 \\
Triglyceride (mmol/1) & $1.63(1.27)$ & $1.63(1.26)$ & $1.62(1.12)$ & 0.99 \\
Current smokers $(\%)$ & 37.7 & 23.5 & 39.4 & 0.27
\end{tabular}

BMI, body mass index. plates in a final volume of $25 \mu \mathrm{l}$ with 30 amplification cycles, denaturation at $94^{\circ} \mathrm{C}$ for one minute, annealing at 53 for 1.5 minutes, and extension at $72^{\circ} \mathrm{C}$ for one minute. The products were analysed using capillary electrophoresis (270-HT, Applied Biosystems, North Warrington, Cheshire, UK) at $30^{\circ} \mathrm{C}$ for 15 minutes at $-13 \mathrm{kV}$. Markers at $104,124,184$, and 192 bp from a Hae III digest of pBr322 were included with each sample.

\section{STATISTICS}

Data are presented as mean (SD) for normally distributed data and geometric mean (SD) for skewed data (triglyceride). Comparisons were performed by $\chi^{2}$ test for categorical variables (current smokers), Mann Whitney U test, and analysis of variance (ANOVA) for continuous variables. Associations between blood pressures and genotypes were assessed by calculation of the median value of the variable and its distribution around this median within genotypes, using $\chi^{2}$ tests to determine significance.

\section{Results}

GENOTYPING AND ALLELE FREQUENCY

In the 245 healthy middle aged men four alleles of the (TAAAA) $)_{n}$ repeat polymorphism in the fibrillin-1 gene were recognised at 181 , 171,166 , and $161 \mathrm{bp}$, identified as alleles 1,2 , 3 , and 4 respectively. The length of the smallest allele (4) was confirmed by DNA sequencing. These four alleles gave rise to only three common genotypes, for which the capillary electrophoretic analysis is shown in fig 1. The frequency of these three genotypes was $2-2$ (157, 64\%), 2-3 (38, 16\%), and 2-4 (37, 15\%). The frequency of the rare genotypes observed was $1-1(1,0.4 \%), 1-2(4,2 \%), 3-3(4,2 \%)$, 3-4 $(1,0.4 \%)$, and $4-4(3,2 \%)$. Since capillary electrophoresis has seldom been used for genotyping, the genotyping was confirmed by gel electrophoresis in 24 cases. There were no discordant results.

\section{ASSOCIATION BETWEEN BLOOD PRESSURE AND} FIBRILLIN GENOTYPE IN HEALTHY MIDDLE AGED MEN

This analysis was restricted further to the three common fibrillin-1 genotypes, a total of 232 individuals. In the search for an association between fibrillin-1 genotype and blood pressure it was thought important to exclude any individuals on drug treatment which could influence blood pressure (for example, $\beta$ blockers, calcium antagonists, diuretics, or angiotensin converting enzyme inhibitors). Therefore 11 individuals (of 2-2 or 2-3 genotype) were excluded from further analyses. The distribution of fibrillin-1 genotypes according to median systolic, diastolic, and pulse pressure for each group and the mean pressure for each genotype for the remaining 221 men is shown in table 1 . Systolic and pulse pressures all show the same trend: mean pressures were lowest for individuals of 2-4 genotype and highest for individuals of 2-3 genotype. Individuals with the most common genotype (2-2) had intermediate pressures. The association was stronger for pulse pressure than for systolic 
pressure. Individuals of 2-4 genotype tended to have below median pulse pressures, individuals of 2-2 genotype had pulse pressures distributed around the median, and individuals of 2-3 genotype tended to have above median pulse pressures $(p=0.003)$. There was no association between fibrillin-1 genotype and mean arterial pulse pressure, age, body mass index, plasma cholesterol or triglyceride concentration, or current smoking status (table 2).

\section{Discussion}

Fibrillin-1, "the Marfan gene", encodes a 350 $\mathrm{kDa}$ protein found in the elastic fibrils of normal aorta, which has been considered responsible for the structural function of microfibrils. ${ }^{11}$ In the healthy middle aged men described here the frequency of a fibrillin-1 genotype, detected using a four allele polymorphism, was similar to that reported in a sample of healthy American individuals, with more than $90 \%$ of the sample showing only three genotypes. ${ }^{8}$ The present study was initiated following our observation of an association between blood pressure (systolic and pulse pressures) and variation in the fibrillin-1 gene in patients with abdominal aortic aneurysm. ${ }^{6}$ These aneurysm patients were elderly with indistensible aortas, and both hypertension and familial or genetic factors could have contributed to the risk of developing an abdominal aortic aneurysm. ${ }^{12}$ Moreover, it has been suggested that a GLY/SER mutation in an epidermal growth factor domain of fibrillin is a risk factor for a similar disorder, thoracic aortic aneurysm. ${ }^{13}$ For all these reasons the associations between fibrillin-1 genotype and blood pressure observed in aneurysm patients might have reflected only genetic selection or stratification. To exclude this possibility it was important to demonstrate similar associations in the general population.

The sample chosen comprised healthy middle aged men, aged 50-61 years, selected from a general practice register. In these men, the association between mean systolic blood pressure and fibrillin-1 genotype was only of borderline significance ( $p=0.10$, ANOVA). Similarly, the association between mean pulse pressure and fibrillin-1 gentotype was of borderline significance $(\mathrm{p}=0.08$, ANOVA). However, there was a significant association between variation in the fibrillin-1 gene and the distribution of pulse pressure, the $15 \%$ of the population with 2-3 genotype having the highest pulse pressures, those with the very common 2-2 genotype having intermediate pressures, and the $15 \%$ of the population with 2-4 genotype having the lowest pulse pressures. Pulse pressure is calculated, not measured, and the numbers of patients with 2-3 and 2-4 fibrillin-1 genotypes are small compared with those of 2-2 genotype.

The elastic recoil of the aorta diminishes with aging, ${ }^{34}$ which may be a consequence of the vascular remodelling with increased depo- sition of polar glycoproteins and proteins other than elastin. ${ }^{3}$ In middle aged men, the distensibility of the abdominal aorta has been reduced by half compared with younger men (20 to 30 years). ${ }^{4}$ Similar loss of distensibility in the proximal (thoracic aorta) will limit the ability of this segment of the aorta to accommodate the stroke volume output from the heart, and contribute to the increase in both systolic blood pressure and arterial pulse pressure observed with aging. Changes in aortic distensibility and diameter also will modify reflected pressure waves along the aorta and contribute to increasing pulse pressure. ${ }^{14}$ Polymorphic variation in the genes encoding the proteins involved in the age dependent vascular remodelling in the aorta could influence the turnover of these proteins and hence the elastic properties of the aortic wall and the development of hypertension. Fibrillin-1 is a strong candidate gene.

The findings reported here are the first indication of an association between fibrillin-1 genotype and blood pressure in the aging population. We suggest that the mechanism underlying the association between variation in the fibrillin-1 gene and pulse pressure arises from modulation of the elastic properties of the aorta and other large arteries in response to aging.

We thank the British Heart Foundation and Charing Cross Hospital Trustees for research support.

1 Dubay C, Vincent M, Samani NJ, Hilbert P, Kaiser MA, Beressi JP, et al. Genetic determinants of diastolic and pulse pressure map to different loci in Lyon hypertensive rats. Nature Genet 1993;3:354-7.

2 Deng AY, Dene H, Rapp JP. Mapping of a quantitative trait locus for blood pressure on rat chromosome 2.7 Clin Invest 1994;94:431-6

3 Spina M, Garbisa S, Hinnie J, Hunter JC, SerafiniFracassini A. Age related changes in composition and mechanical properties of the tunica media of the upper thoracic human aorta. Arteriosclerosis 1983;3:64-74.

4 MacSweeney STR, Young G, Greenhalgh RM, Powell JT. Mechanical properties of the aneurysmal aorta. Br F Surg 1992;79:1281-4.

5 Bader H. Importance of the gerontology of elastic arteries in the development of essential hypertension. Clin Physiol Biochem 1983;1:36-56.

6 MacSweeney STR, Skidmore C, Turner RJ, Sian M, Brown L, Henney AM, et al. Unravelling the familial tendency to aneurysmal disease: popliteal aneurysm, hypertension and fibrillin genotype. Eur f Vasc Endovasc Surg 1996;12:162-6.

7 Lee B, Godfrey M, Vitale E, Hori H, Mattei M-G, Sarfarazi $M$, et al. Linkage of Marfan syndrome and a phenotypically related disorder to two different fibrillin genes. Nature 1991;352:330-4.

8 Periera L, Levran O, Ramirez F, Lynch JR, Sykes B, Pyeritz $\mathrm{RE}$, et al. A molecular approach to the stratification of cardiovascular risk in families with Marfan's syndrome. $N$ Engl f Med 1994;331:148-53.

9 Nakamura Y, Leppert M, O'Connell P, Wolff R, Holm T, Culver M, et al. Variable number of tandem repeat (VNTR) markers for human gene mapping. Science 1987;235:161622 .

10 Miller GJ. Risk of thrombosis and the prethrombotic state. In: Woodford FP, Davignon J, Sniderman A, eds. In: Woodford FP, Davignon J, Sniderman A,

11 Zhang H, Hu W, Ramirez F. Developmental expression of fibrillin genes suggests heterogeneity of extracellular microfibrils. F Cell Biol 1995;29:1165-76.

12 Abdominal aortic aneurysm. Grand round. Lancet 1993; 341:215-20.

13 Francke U, Berg MA, Tynan K, Brenn T, Lin W, Aoyama T, et al. A gly 1127 ser mutation in an EGF-like domain of the fibrillin-1 gene is a risk factor for ascending aortic aneurysm and dissection. Am f Hum Genet 1995;56:128796.

14 Safar ME. Pulse pressure in essential hypertension: clinical and therapeutic implications. $\mathcal{F}$ Hypertens 1989;7:769-76. 